\title{
COVID-19 and our canine companions: is biodetection a viable alternative?
}

\author{
Jared Robinson, Alexandra Leclézio, Indrajit Banerjee* \\ Sir Seewoosagur Ramgoolam Medical College, Belle Rive, Mauritius
}

\section{LETTER TO THE EDITOR}

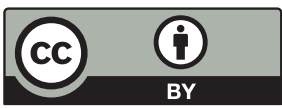

This work is licensed under a Creative Commons Attribution 4.0 Unported License.

Currently, real-time reverse transcription-PCR (RT-PCR) assays is the molecular test of choice for the etiologic diagnosis of SARS-CoV-2 infection, whilst supplemental tools include anti-body-based techniques. Carrying out PCR testing is time consuming and expensive; a single RT-PCR test kit may cost over 100 USD, while setting up a highly complex diagnostic and processing lab, including staff training, requires more than 15,000 USD. ' The lack of availability and accessibility of these tests makes them difficult to use on a large scale, especially in developing countries. Now, more than ever, the world is in desperate need of a sensitive, rapid, inexpensive, non-invasive method of screening large numbers of asymptomatic people.

Volatiles (infectious micro-organism specific organic compounds released in breath, sweat and urine) could be used as bio-markers for detection of specific viruses. $^{2}$ Volatile Organic Compounds (VOCs) of different microorganisms have specific odours, and have been detected by Trained Dogs, in the detection of malaria and Influenza. ${ }^{3}$ Furthermore, in Germany, preliminary findings show that trained scent dogs were able to differentiate SARS-CoV-2 infected individuals from non-infected individuals. ${ }^{4}$ These studies showed that dogs could identify malaria and influenza with a degree of sensitivity and specificity within the World Health Organization's criteria for the selection of rapid tests. ${ }^{5}$ This could prove to be extremely useful for mass COVID-19 screening, since the lack of symptoms render it undetectable by numerous screening tests.

This would be a good application for countries like Nepal which are developing, for example, screening travellers at airports is an useful method to prevent imported COVID-19 infections, thus reducing the need to enforce quarantine measure on incoming travellers. This would reduce the cost of quarantine on governmental hospitals as well reduce spread of the virus and the costs of treatment. Since Nepal has a large population, bio-detection dogs would prove to be a useful screening method as they can screen up to 250 people in an hour. ${ }^{5}$

\section{CONCLUSION}

It is imperative that this screening method is used in Nepal, as it will save the lives of many. Because of it's attributes, this would be a good COVID-19 screening method for use in Nepal. Nepal could benefit from a rapid, accurate, mobile, inexpensive screening method such as Bio-Detection of Volatiles by trained dogs.

\section{REFERENCES}

1. Ramdas K, Darzi A, Jain S. 'Test, re-test, re-test': using inaccurate tests to greatly increase the accuracy of COVID-19 testing. Nat Med. 2020 Jun;26(6):810-811. https://doi:10.1038/ s41591-020-0891-7.

2. Shirasu M, Touhara K. The scent of disease: volatile organic compounds of the human body related to disease and disorder. J Biochem. 2011 Sep;150(3):257-66. https://doi:10.1093/jb/ mvr090.

3. Guest $C$, Pinder $M$, Doggett $M$, et al. Trained dogs identify people with malaria parasites by their odour. Lancet Infect Dis. 2019 Jun;19(6):578-580. https://doi:10.1016/S14733099(19)30220-8

4. Jendrny P, Schulz C, Twele F, et al. Scent dog identification of samples from COVID-19 patients - a pilot study. BMC Infect Dis. 2020 Jul 23;20(1):536. https://doi:10.1186/s12879-02005281-3.

5. Jones RT, Guest C, Lindsay SW, et al. Could bio-detection dogs be used to limit the spread of COVID-19 by travellers? J Travel Med. 2020 Aug 12:taaa131. https://doi:10.1093/jtm/taaa131

*Corresponding Author | Dr. Indrajit Banerjee, Associate Professor Department of Pharmacology, Sir Seewoosagur Ramgoolam Medical College, Belle Rive, Mauritius | Email: indrajit18@gmail.com 